International Journal of Information Sciences and Techniques (IJIST) Vol.2, No.4, July 2012

\title{
DESIGN AND DEVELOPMENT OF A LOW-COST Microcontroller Based Single Phase WATER-PUMP CONTROLLER
}

\author{
M.A.A. Mashud ${ }^{1 *}$, M.A.A. Tariq ${ }^{1}$, M. Shamim Hossain ${ }^{2}$ and Md. Serajul Islam ${ }^{3}$ \\ ${ }^{1}$ Department of Applied Physics, Electronics and Communication Engineering \\ Islamic University, Kushtia-7003, Bangladesh \\ ms.mashud@yahoo.com, tariq_iuk@yahoo.com \\ ${ }^{2}$ Department of Computer Science and Engineering, Islamic University, Kushtia-7003, \\ Bangladesh \\ shamimmalitha@yahoo. com \\ ${ }^{3}$ Department of Medical Physics and Biomedical Engineering, Gono Bishwabidyalay, \\ Savar, Dhaka, Bangladesh \\ i_serajul_islameyahoo.com
}

\begin{abstract}
A microcontroller based advanced technique was designed and developed to protect the house hold appliances, such as water-pump from fluctuation of line voltage. This device was tested with upper and lower cutoff voltages set at $\pm 10 \%$ of the normal supply voltage $(220 \mathrm{~V}, A C)$ and with an over-load current up to 10A. The current sensor's output was monitored by the PIC12F675 microcontroller and used analog to digital protocol. A ' $C$ ' language program was developed to control the function of microcontroller, using PCWH compiler.
\end{abstract}

\section{KEYWORDS}

PIC12F675 microcontroller, Over-load, Hall-sensor, Single-phase and PCWH compiler

\section{INTRODUCTION}

The fluctuations of line voltage in our country sometimes causes serious problem to sophisticated electrical and electronic equipment. Quite often it happens that the main supply goes off, remains for a short period and then restores itself. Particularly during the storm season, it is observed that the line voltages of some phases go down to a low level and remain at the level for several hours, until some corrective measures are taken by the concerned authority. Under such erratic conditions of line voltage, in our country re-wireable fuse or miniature circuit breaker are normally used to protect different households from under and over-voltage conditions is explained in S. Islam et.al, [1]. Considering this factor, a protective device like over-voltage and over-current with time delay unit has been developed for ensuring a dependable and an effective protection to small households in year 1992 that explained in S. Islam et.al, [2]. This system is very slow and designed in transistor based. For the better performance another device has been

DOI : $10.5121 /$ ijist.2012.2405 
developed to protect households in year 1994 which explained in S. Islam et.al, [3]. The operation speed of this device is better but it was designed in transistor based. In year 2011, another attractive device has been developed for single-phase line to protect electronic appliances from over-load which explained in M.A.A. Mashud et.al, [4]. In this design transistor module has been replaced by IC module and use Hall Effect principle.

We developed an alternative approach: microcontroller based single phase water-pump controller. In our design all electronic components and ICs has been replaced by using PIC12F675 microcontroller. Easily available components and simple circuitry, the system should be beneficial in providing low-cost.

\section{SYSTEM DESIGN}

The system is divided into four main parts: low-voltage power supply, current sensing circuit, microcontroller unit and relay driver circuit. In this unit, an alternating voltage $(220 \mathrm{~V})$ was applied at the input of a step-down transformer that gave $12 \mathrm{~V}$ output which was further fed to four diodes $1 \mathrm{~N}$ 4001(A bridge Rectifier) which gave 12V DC output which was made ripple free by the use of filter capacitors. This filtered $12 \mathrm{~V}$ DC was then fed to a relay unit for its operation. The majority of components required $5 \mathrm{~V}$ for their operation. For this purpose, LM 7805 (3terminal voltage regulator) was employed that converted $12 \mathrm{~V}$ DC into $5 \mathrm{~V}$ DC. In this way, all the components needing $5 \mathrm{~V}$ for their operation were connected to the output of IC 7805. This voltage was fed at pin 1 of the PIC12F675 microcontroller as a supply voltage. Pin 7 of the microcontroller were control input which coupled with current sensor. A resistance of $4.7 \mathrm{~K} \Omega$ was soldered between pin 1 and pin 4 which denoted by $R_{1}$. Also a resistance of $100 \Omega$ was soldered at the output (pin 5) of the microcontroller which denoted by $\mathrm{R}_{2}$. The AC output of the current sensor was converted into DC as the analog input of the microcontroller. LED1 and LED2 were used to indicate the over-voltage low-voltage level respectively. The block diagram and the complete circuit diagram of the developed system are depicted in Figure 1 and Figure 2 respectively.

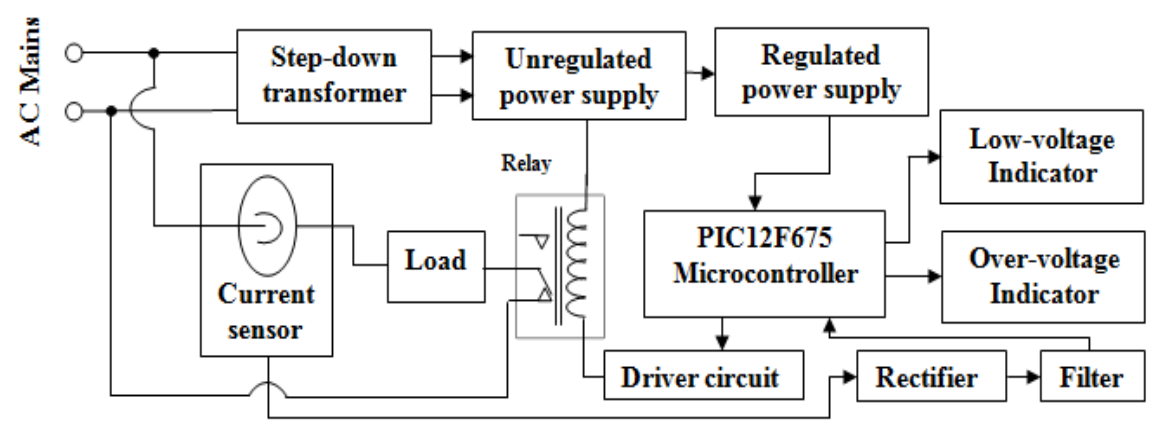

Figure 1: Block diagram of the developed system 


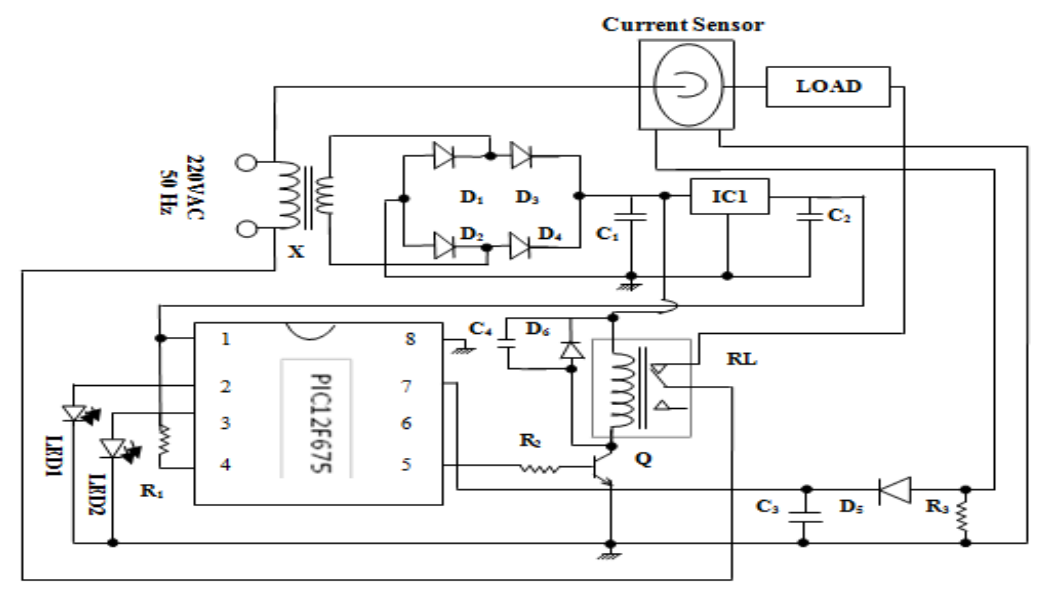

Figure 2: Complete circuit diagram of the developed system

\subsection{Low-voltage Power Supply}

The low-voltage power supply circuit consists of transformer $\mathrm{X}, \mathrm{D}_{1}, \mathrm{D}_{2}, \mathrm{D}_{3}, \mathrm{D}_{4}, \mathrm{C}_{1}, \mathrm{C}_{2}$, and IC1 [5]. The output of the IC1 is $+5 \mathrm{~V} \mathrm{DC}$. The capacitor $\mathrm{C}_{2}$ is connected across the output of IC1 to eliminate the high frequency noise.

\subsection{Current Sensing Circuit}

The current sensing circuit is consisting of current sensor, $\mathrm{R}_{3}, \mathrm{D}_{5}$ and $\mathrm{C}_{3}$. One of the connecting wires of the load passes through the current sensor without interrupting it to the coil. When current passes through the load, an ac voltage is induced across the coil of the sensor. The sensor is designed in such a way that the voltage developed across the coil is proportional to the amount of load current. The output of the current sensor is rectified and filtered.

\subsection{Microcontroller (PIC 12F675) unit}

This powerful (200 nanosecond instruction execution) and easy-to-program (only 35 single word instructions) CMOS FLASH-based 8-bit microcontroller packs Microchip's powerful Programmable Interface Controller (PIC) architecture into a 8-pin package [6] and is compatible with the PIC12F675 and PIC12F629 devices. The PIC microcontroller is a Multipoint Control Unit (MCU) that is used in this project. MCU measures the period of the input signal and it converts into a digital voltage data. The PIC's Console Command Processor (CCP) which can also detect rising or falling edges every four or 16 pulses. PIC12F675 features 128 bytes of electrically erasable programmable read-only memory (EEPROM) data memory, self programming, 4 channels of 10-bit Analog-to-Digital (A/D) converter [7, 8]. All of these features make it ideal for an advanced level A/D applications in automotive, industrial appliances and consumer applications.

\section{Features}

- High-Performance RISC CPU

- Only 35 single word instructions to learn

- All instructions are single cycle $(1 \mu \mathrm{s})$ except for program branches 
International Journal of Information Sciences and Techniques (IJIST) Vol.2, No.4, July 2012

- Operating speed: DC - 20MHz clock input

- 1 k Bytes Flash Program Memory

- 64 Byte RAM Data Memory

- 128 Byte EEPROM Data Memory

- In-circuit serial programming

\section{Peripherals Features}

- One 8-bit timer/counter (TMR0) with 8- bit programmable prescalar

- One 16 bit timer/counter (TMR1)

-10-bit, 4-channel Analog-to-Digital converter

- High current sink/source for direct LED driver

- One Analog Comparator

-Watchdog Timer (WDT) with independent oscillator for reliable operation

\section{Special Microcontroller Features}

- 100,000 erase/write cycle Enhanced FLASH program memory

- 1,000,000 erase/write cycle Data EEPROM memory typical

- Power saving SLEEP mode

- Programmable code protection

- Selectable Oscillator Options

- Self-reprogrammable under software control

- Flash/Data EEPROM Retention: > 40 years

\section{CMOS Technology}

- Low power, high speed CMOS FLASH technology

- Fully Static Design

- Low Power Consumption

\section{I/O and Packages}

- $6 \mathrm{I} / \mathrm{O}$ pins with individual direction control

-8-pin DIP

\subsection{Relay Driver Circuit}

The relay driver circuit consists of $\mathrm{R}_{2}, \mathrm{C}_{4}, \mathrm{D}_{6}$, transistor $\mathrm{Q}$, and relay RL. The transistor $\mathrm{Q}$ acts as emitter follower [9], the output of which drives the relay RL. Diode $\mathrm{D}_{4}$ across the relay coil protects the transistor Q from the back EMF induced in the relay coil during breaks. The load current depends on the contact rating of the relay.

\section{SySTEM PROGRAM}

The software was divided into different sub routines and main routines. The compiler PCWH was used to develop the software [10]. 


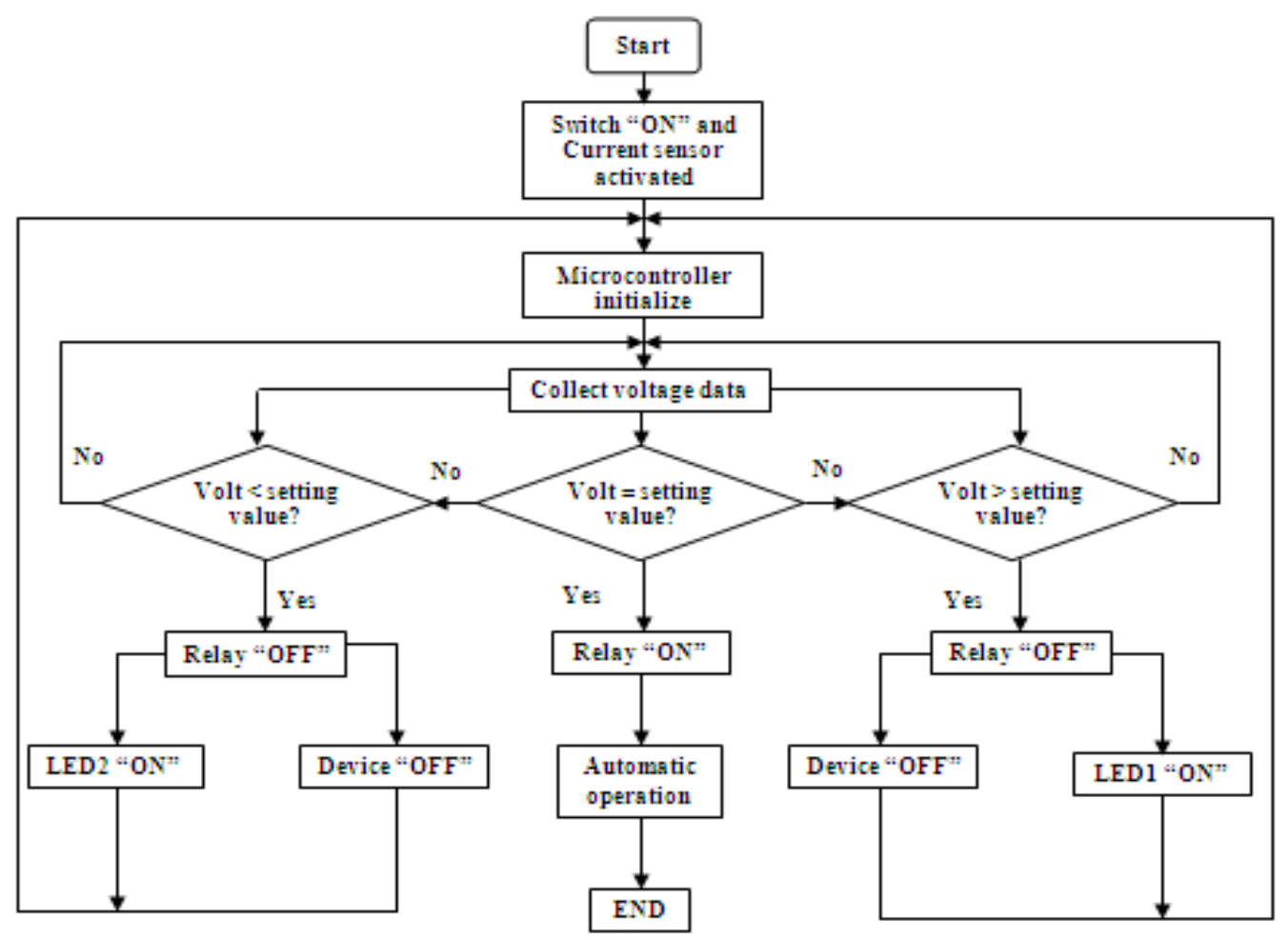

Figure 3: Flow chart of the system program

The system program and flow chart is depicted in Figure 3 that shows the interaction of line voltage and water-pump via microcontroller. When the system is activated, it will invoke the microcontroller to reset all hardware devices in a normal and controlled mode. The current sensor will then start to sensing the current and produce the corresponding voltage data that records the microcontroller. If the collected voltage data is less than the setting value, the relay turns off; LED2 indicates light and the device off. Also, if the collected voltage data is high than the setting value, the relay turns off, LED1 indicates light and the devices also off. Otherwise, the device will be switched $\mathrm{ON}$ automatically and the system will complete automatic operation.

\section{RESULTS AND DISCUSSION}

The system was successfully developed and its performance was strong. The system employed a local, low-cost PIC12F675 microcontroller. To avoid the complex comparator and A/D converter circuit, we used an internal comparator and A/D converter for the microcontroller. Furthermore, the internal frequency successfully avoided the external oscillator circuit. Thus the entire system function depends on the developed software. The circuit design is simple and compact.

The low voltage power supply unit was designed for highly regulated output to bias the microcontroller. In this design we had varied the input ac voltage from 198 volt to 248 volt but the output was remain constant at 5 volt DC. The output waveform of this unit is depicted in Figure 4. 
International Journal of Information Sciences and Techniques (IJIST) Vol.2, No.4, July 2012

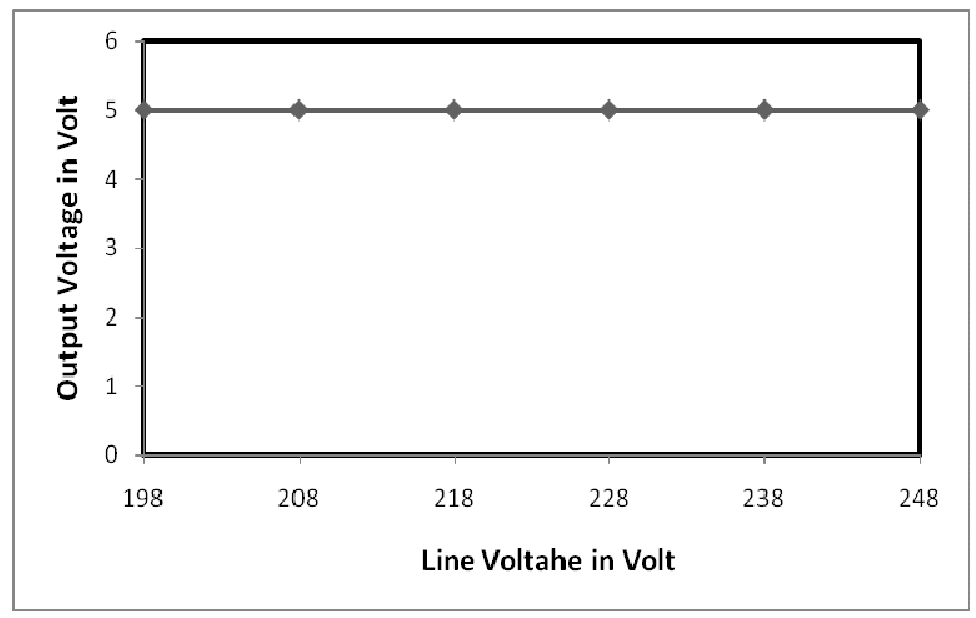

Figure 4: Output of the low-voltage power supply

The current sensor with its circuitry was designed in such a way, that it provided 2.5 volt dc to 5 volt $\mathrm{dc}$ for the line voltage fluctuation between $\pm 10 \%$ of 220 volt. The output level of this unit is shown in Figure 5.

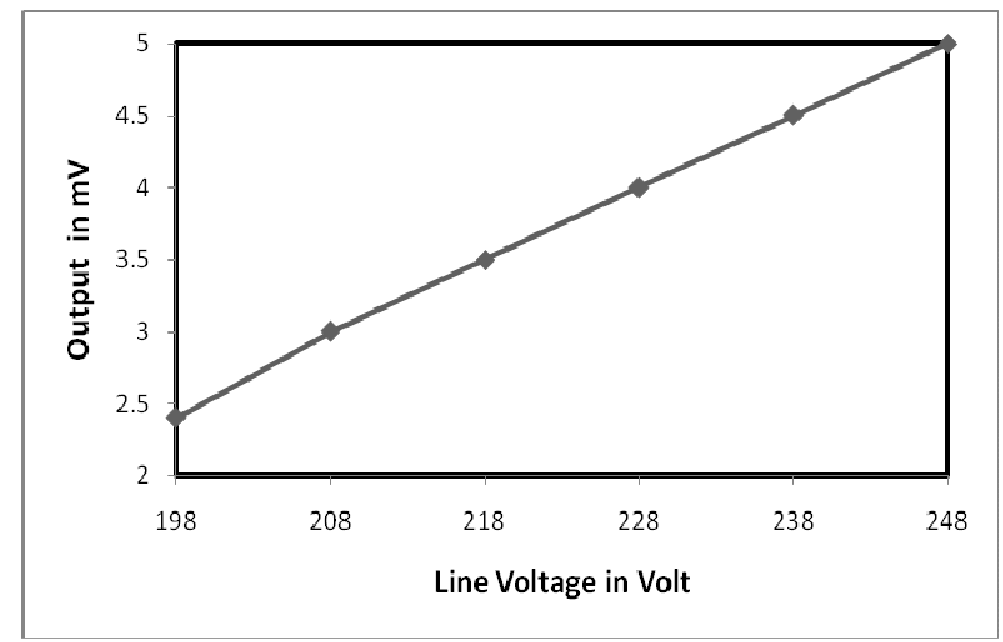

Figure 5: DC output level of the current sensor

The performance of the developed system was studied by comparing its accuracy, reliability and cost. The working of this device was similar to that on which M.A.A. Mashud et. al., worked [4]. A series of large-scale laboratory experiments were conducted with the help of this unit, with satisfactory results. This unit was used with single phase water-pump which had sound stability, operation speed and accuracy.

The comparative study of the developed system in terms of features and accuracy with the conventional system is depicted in table 1 . It can be seen from Table 1 that the developed system has very good accuracy. The cost of the system which is significantly lower compared to other systems with similar features is another advantage of this system. 
International Journal of Information Sciences and Techniques (IJIST) Vol.2, No.4, July 2012

Table 1. Comparisons of the developed system with the conventional system

\begin{tabular}{|l|c|c|c|}
\hline & $\begin{array}{l}\text { Transistor Based } \\
\text { System [3] }\end{array}$ & $\begin{array}{l}\text { IC Based } \\
\text { System [4] }\end{array}$ & Developed System \\
\hline Module & Transistor & IC & Microcontroller \\
\hline Operation Type & Analog & Analog & Digital \\
\hline Accuracy & $\pm 0.5 \%$ & $\pm 0.2 \%$ & $\pm 0.05 \%$ \\
\hline Operation Speed & Slow & Medium & High \\
\hline Circuit design & Complex & Simple & Very Simple \\
\hline
\end{tabular}

\section{CONCLUSION}

The developed system was put under a series of tests for ascertaining its performance as a protective device and very satisfactory results were obtained. When the load current exceeds $10 \mathrm{~A}$, the load was turned off. The upper and lower level responses of this device were also found to be sufficiently quick, so that the safety of the equipment protection by the device under any undesired transient condition of the main supply was ensured. This device had a very high sensitivity. It was also simple in design, reliable in operation and cost competitive with any other product available in the market. From the above analysis, it is concluded that this device can easily protect electrical appliances against fluctuation of line voltages and over load current.

\section{REFERENCES}

[1] M. S. Islam and M. A. Zulquarnain, (1991), Journal of the Bangladesh Electronics Society, pp 31.

[2] M. S. Islam and M. Kamruzzaman, (1992), Journal of the Bangladesh Electronics Society, Vol.2,No. 1 , pp 29.

[3] S. Islam, (1994) Nuclear Science and Applications, Vol. 3, No. 2.

[4] M.A.A. Mashud, E. Haque and S. Islam, (2011) Jahangirnagar Physics Studies, Vol. 17, pp 151 ISSN 1999-6632.

[5] V. K. Mehta and R. Mehta, (2007) Principles of Electronics, S. Chand \& Company Ltd. New Delhi, pp 438.

[6] http:/ww1.microchip.com/downloads/en/devicedoc/41190c.pdf, 2

[7] M. A. Mazidi, R.M. Kinlay \& D. Causey, (2008) "PIC Microcontroller", Prentice Hall Inc.,pp 24.

[8] J. B. Peatman, (1997) "Design with PIC microcontroller", Prentice Hall Inc., pp 2

[9] B.L. Theraja and A.K Theraja, (2002) A Textbook of Electrical Technology, S. Chand \& Company Ltd. New Delhi, pp 2105.

[10] PCWH Compiler®IDE version 3.43, www.ccsinfo.com

\section{Authors}

Md. Abdullah-Al-Mashud (M. A. A. Mashud) was born on Nov.15, 1980 in kushtia, Bangladesh. He received the B.Sc (Hons) degree and M.Sc degree in Applied Physics, Electronics and Communication Engineering (APECE) from Islamic University, Kushtia, Bangladesh in 2003 and 2004 respectively. He works as a lecturer in the department of APECE, Islamic University, Bangladesh. His current interest is microprocessor / microcontroller applications in control, automation, medical instruments, environmental monitoring, low cost electronic systems, Medical Image: Segmentation, Processing and assessment. His work has produced 08 peer-reviewed scientific International and National Journal papers. He has published 04 papers in

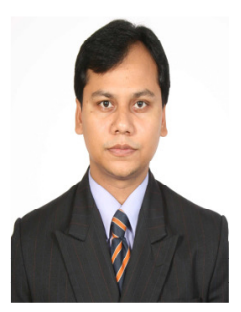


National and International Conferences.

Prof. Md. Serajul Islam was born in Panchagar, Bangladesh. He received the M.Sc degree in Physics from Rajshahi University, Bangladesh. He was a Chief Scientific Officer and Director in the Institute of Electronics, AERE, Atomic Energy Commission, Savar, Bangladesh. Now he is a Professor in the department of Medical physics and Biomedical Engineering, Gono Bishwabidyalay, Saver, Bangladesh. His work is design, development and analysis of electronic instruments and reactor control. His work has produced nearly 45 peer-reviewed scientific papers and 02 patents.

M. A. A. Tariq was born on Dec.14, 1989 in Meherpur, Bangladesh. He a B.Sc (Hons) final year student in the department of Applied Physics, Electronics and Communication Engineering (APECE), Islamic University, Kushtia, Bangladesh.. His current interest is automation and microcontroller based system design.
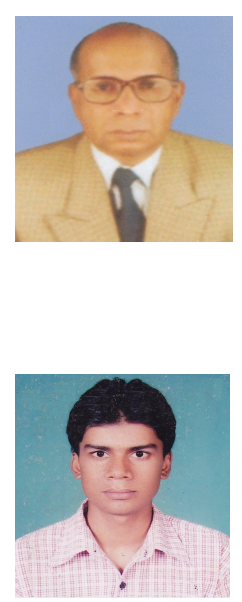

M. Shamim Hossain was born on Oct. 21, 1982 in kushtia, Bangladesh. He received the B.Sc (Hons) degree and M.Sc degree in Computer Science and Engineering (CSE) from Islamic University, Kushtia, Bangladesh in 2006 and 2007 respectively. He works as a lecturer in the department of CSE, Islamic University, Bangladesh. His current interest is microprocessor / microcontroller based system design and wireless networking. His work has produced 06 peer-reviewed scientific International Journal papers

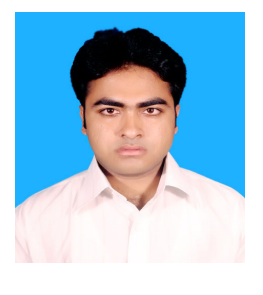

